\title{
Efficient production of negative hydrogen ions in RF plasma by using a self-biased grid electrode
}

\author{
Kohgi Kato and Satoru Iizuka \\ Department of Electrical Engineering, Graduate School of Engineering, Tohoku University, \\ Aza Aoba 6-6-05, Aramaki, Aoba-ku, Sendai 980-8579, Japan
}

\begin{abstract}
:
Volume production of negative hydrogen ions is established efficiently in a pure hydrogen RF discharge plasma by using a self-biased grid electrode for production of low electron-temperature and high density plasma. Using this electrode both high and low electron temperature plasmas are produced in the regions separated by the grid electrode in the chamber, in which the electron temperature in the downstream region is controlled by the mesh size and plasma production parameters. The production rate of negative ions depends strongly on the electron temperature varied by the RF input power and hydrogen pressure. In the case of the grid electrode with the 5 mesh/in., the negative hydrogen ions are produced effectively in the downstream region in the hydrogen pressure range of $0.9-2.7 \mathrm{~Pa}$. In addition, the production rate of the negative ion $\mathrm{H}^{-}$raises from $62 \%$ to $87 \%$ at $0.9 \mathrm{~Pa}$ by changing the RF power from $20 \mathrm{~W}$ to $80 \mathrm{~W}$.

Keywords: negative hydrogen ion, self-biased grid electrode, capacitively-coupled RF discharge, low electron temperature.
\end{abstract}

\section{INTRODUCTION}

In reactive plasmas widely used for material processing, electron energy distribution would give a big effect on the products obtained, because excitation and desociation are determined by the electron energy. When electrons distribute apploximately like Maxwelian, the average electron energy is expressed by the electron temperature $T_{\mathrm{e}}$. Therefore, the control of $T_{\mathrm{e}}$ is of crucial importance for finding the best conditions of the chemical reactions necesary for the material processing [14]. In general, however, it is difficult to control $T_{\mathrm{e}}$ in weakly-ionized discharge plasmas, although several methods have been reported for the $T_{\mathrm{e}}$ control [1-9].

In our method using a mesh-grid [5], $T_{\mathrm{e}}$ is decreased by almost two orders of magnitude, being accompanied by an increase in electron density $n_{\mathrm{e}}$. The grid-bias method, applied to RF silane plasma, revielded a good reproducible yield of hydrogenated amorphous silicon (a-Si:H) films with high drift mobilty [10]. We also applied this method to negatve-ion production in RF hydrogen plasma, where the negative hydrogen ion production was enhanced by the decrease in $T_{\mathrm{e}}$ [11]. In the case of RF discharge plasma, the grid bias method was found to be quite suitable for optimizing the plasma conditions for negative ion production, compared with the magnetic filter method $[12,13]$.

It is pointed out that the grid-bias method is inappropriate for reactive plasmas, in which grid is often covered by thin insulating films like hydrogenated amorphous and diamond-like carbon films. However, we have already demonstrated that the grid bias method can still control $T_{\mathrm{e}}$ over a wide range [9]. In fact, high-quality diamond was produced in RF discharge $\mathrm{CH}_{4} / \mathrm{H}_{2}$ plasmas [14], where $T_{\mathrm{e}}$ was decreased by varying mechaniclly the length of slits in plasmas [8]. In the case of Argon plasma, $T_{\mathrm{e}}$ was widely controlled by almost one order of magnitude by changing the mesh size of the grid electrode.

It is well known that negative hydrogen ions $\mathrm{H}^{-}$are generated by the next two stages. In the first stage, high energy electrons $\mathrm{e}_{h}$ with $T_{\mathrm{e}}$ more than $20 \mathrm{eV}$ excite $\mathrm{H}_{2}$ molecules to generate vibrationally excited molecules $\mathrm{H}_{2}^{*}(\mathrm{v})$ via the reaction $\mathrm{H}_{2}+\mathrm{e}_{h} \rightarrow \mathrm{H}_{2}^{*}(v)+\mathrm{e}$. In the second stage, low energy electrons $\mathrm{e}_{l}$ with $T_{\mathrm{e}} \sim 1 \mathrm{eV}$ collide with $\mathrm{H}_{2}^{*}(\mathrm{v})$ for the production of $\mathrm{H}^{-}$via dissociative attachment collisions reaction $\mathrm{H}_{2}^{*}(v)+$ $\mathrm{e}_{l} \rightarrow \mathrm{H}^{-}+\mathrm{H}$ [15]. In the second stage, however, very low energy electrons with $T_{\mathrm{e}}<0.5 \mathrm{eV}$ have too small energy to cause the above reaction effectively [16]. Note that negative ions are easily destroyed by the collisions with warm electrons. Therefore, it is necessary to generate $\mathrm{e}_{h}$ and $\mathrm{e}_{l}$ in separate regions, and keep them isolated each other. Our device using the self-biased electrode is very suitable to genarate negative ions efficiently because $\mathrm{e}_{h}$ and $\mathrm{e}_{l}$ are isolated in two regions by the self-biased grid electrode.

In this paper, we propose a new method using a self-biased grid electrode for a production of the RF discharge hydrogen plasma. Here, 
negative hydrogen ions are produced by controlling $T_{\mathrm{e}}$ in a steady-state capacitively-coupled RF plasma [17]. By using a grid electrode with mesh size of 5 mesh/inch, we have succeeded in generating $\mathrm{H}^{-}$ ions efficiently in the RF plasma under the $\mathrm{H}_{2}$ pressure range of $0.9-2.7 \mathrm{~Pa}$.

\section{EVALUATION OF NEGATIVE ION DENSITY}

First, let us estimate negative ion density $n^{-}$by measuring negative and positive saturation currents $I^{-}$and $I^{+}$of a Langmuir probe in hydrogen plasmas. Here, we assume that the ions of positives and negatives are monovalent ions, $\mathrm{H}^{+}$and $\mathrm{H}^{-}$, respectively, as confirmed experimentally below. In addition, by supposing that $M_{+}, M_{-}, T_{-}$and $T_{\mathrm{e}}$ are mass of positive ion, mass of negative ion, temperature of negative ion, and electron temperature, respectively, the saturation currents $I^{-}$and $I^{+}$are expressed by the next eqations [18-20].

$$
\begin{gathered}
I^{-}=\mathrm{eS}\left[n_{\mathrm{e}} \sqrt{\frac{k T_{\mathrm{e}}}{2 \pi \mathrm{m}_{\mathrm{e}}}}+n^{-} \sqrt{\frac{k T_{-}}{2 \pi M_{-}}}\right] \\
I^{+}=\mathrm{eSn} n^{+} \sqrt{\frac{k T_{\mathrm{e}}}{2.72 M_{+}}}
\end{gathered}
$$

Here, the factor 2.72 is the Napier's constant, coming from a density difference between the sheath edge and the plasma region. From Eqs. (1), (2) and $n^{+}=n_{\mathrm{e}}+n^{-}$(Condition of the electric charge neutrality) one can obtain the next equation.

$$
\frac{I^{-}}{I^{+}}=\sqrt{\frac{2.72 M_{+}}{2 \pi \mathrm{m}_{\mathrm{e}}}}\left[1-\frac{n^{-}}{n^{+}}\left\{1-\sqrt{\frac{\mathrm{m}_{\mathrm{e}} T_{-}}{M_{-} T_{\mathrm{e}}}}\right\}\right] .
$$

Here, by using $T_{-} \ll T_{\mathrm{e}}$ and $\mathrm{m}_{\mathrm{e}} \ll M_{-}$, the last term in Eq. (3) can be neglected. Therefore, the density ratio of negative to positive ions $n^{-} / n^{+}$is given by the next expression.

$$
\frac{n^{-}}{n^{+}} \cong 1-\frac{I^{-}}{I^{+}} \sqrt{\frac{2 \pi \mathrm{m}_{\mathrm{e}}}{2.72 M_{+}}}
$$

By using positive hydrogen-atom ion mass $M_{+}$, one can obtain the next expression

$$
\frac{n^{-}}{n^{+}}=1-0.0348 \times \frac{I^{-}}{I^{+}}
$$

In the case that different positive hydrogen ion species $\left(\mathrm{H}^{+}, \mathrm{H}_{2}^{+}\right.$and $\left.\mathrm{H}_{3}^{+}\right)$are produced, $M_{+}$must be replaced by weighted average mass $M_{\mathrm{av}}[21]$. However, in the case of $\mathrm{H}_{2}$ plasma as in our experiment, the majority of positive and negative ions are $\mathrm{H}^{+}$and $\mathrm{H}^{-}$, respectively [See Fig. 13 of Ref. 21]. From Eq. (2) positive ion density $n_{\mathrm{i}}=n^{+}$is expressed as follows with a use of $I^{+}=$ $I_{\text {is }}$.

$$
\begin{aligned}
& n_{\mathrm{i}}=n^{+}=\frac{I^{+}}{e S} \sqrt{\frac{2.72 M_{+}}{k T_{\mathrm{e}}}} \\
& =1.50 \times 10^{11} \frac{I_{\mathrm{is}}}{\sqrt{T_{\mathrm{e}}}}\left[\mathrm{cm}^{-3}\right]
\end{aligned}
$$

where the units of $I_{\mathrm{is}}$ and $T_{\mathrm{e}}$ are $\mathrm{mA}$ and $\mathrm{eV}$, respectively. Probe surface area is $S=0.07 \mathrm{~cm}^{2}$.

\section{EXPERIMENTAL SETUP}

Figure 1 shows a schematic of the experimental apparatus. A rectangular electrode with a mesh grid shown in Fig. 2 is placed on a side wall of a grounded cubic chamber of $15 \times 15 \times$ $15 \mathrm{~cm}^{3}$ in volume. The cubic chamber is installed in a stainless steel vacuum chamber of $60 \mathrm{~cm}$ in diameter and $100 \mathrm{~cm}$ in length, which is evacuated to a pressure of $0.3 \mathrm{~Pa}$ by using a rotary pump. Back and side surfaces of this electrode are made of aluminum plates with thickness of $0.5 \mathrm{~mm}$, which are covered with aluminum plates for an electric shielding. Space of $3 \mathrm{~mm}$ exists between the aluminum electrode and the shielding plates. The back area and height of this electrode are $5 \times 5 \mathrm{~cm}^{2}$ and $2 \mathrm{~cm}$, respectively. The mesh electrode of $5 \mathrm{mesh} / \mathrm{inch}$ is made of stainless steel wires of $0.5-\mathrm{mm}$-diam. The area of the mesh grid is $25 \mathrm{~cm}^{2}$. Hydrogen plasma is generated by a 40 $\mathrm{MHz}$ power source in pressure range of $0.7 \sim 3.7$ $\mathrm{Pa}$. RF power $P_{\mathrm{RF}}$ is supplied to the electrode directly through a blocking capacitor (BC) in a range of $20-80 \mathrm{~W}$. As a result, self-bias voltage is induced on the electrode [22, 23]. If necessary, Argon gas is used for obtaining basic parameters of plasma. 


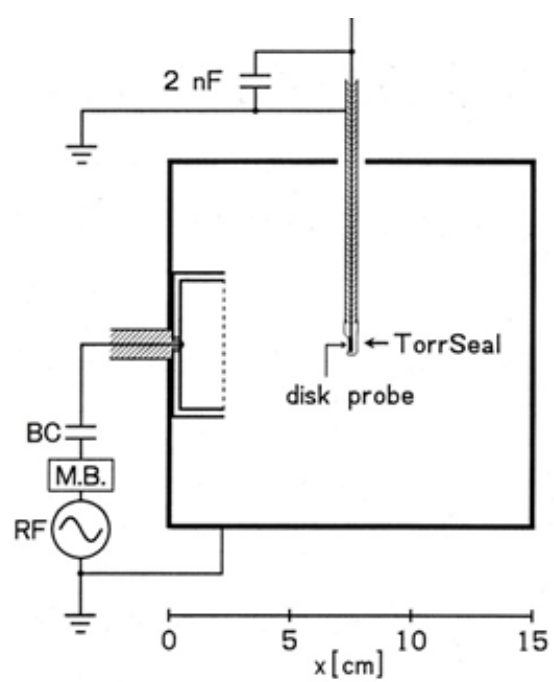

Figure 1. Schematic of the experimental apparatus for an efficient production of negative hydrogen ions in $\mathrm{RF}$ $(=40 \mathrm{MHz})$ discharge plasma.

Plasma parameters are measured by a disc probe of $3 \mathrm{~mm}$ in diameter. One side of the probe is used as a collector of the probe current, and the other surface is covered with Torr Seal which is a sealant made of low vapor pressure resin. Therefore, the surface area of the probe is equal to $0.07 \mathrm{~cm}^{2}$. The values of the dc probe current $I_{\mathrm{p}}$ and voltage $V_{p}$ collected through Source Meter-2400 manufactured by Keithley Instruments are stored on a personal computer, and at the same time, a probe characteristic curve is drawn automatically

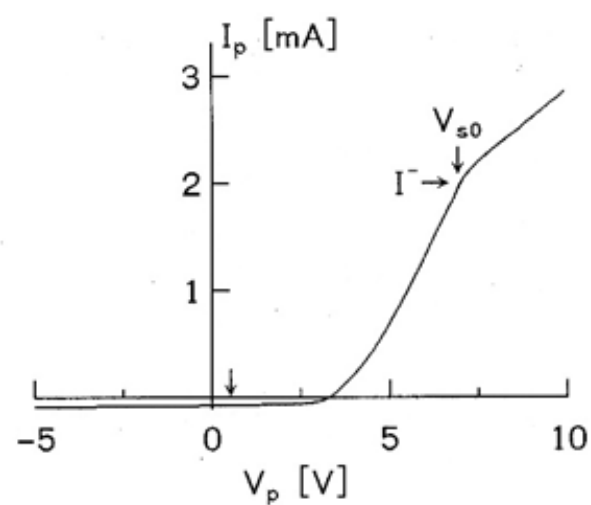

Figure 3. Time-averaged $I_{\mathrm{p}}-V_{\mathrm{p}}$ probe characteristic curve in $\mathrm{H}_{2}$ plasma. $P_{\mathrm{RF}}$ and $P_{\mathrm{H}_{2}}$ are $20 \mathrm{~W}$ and $3.6 \mathrm{~Pa}$, respectively. $x=7.5 \mathrm{~cm}$.

\section{EXPERIMENTAL RESULTS}

Time averaged probe $I_{\mathrm{p}}-V_{\mathrm{p}}$ characteristic curve for pure hydrogen plasma is shown in Fig. 3 in the case of RF power $P_{\mathrm{RF}}=20$ $\mathrm{W}$ and pressure $P_{\mathrm{H}_{2}}=3.6 \mathrm{~Pa}$. The mesh size and

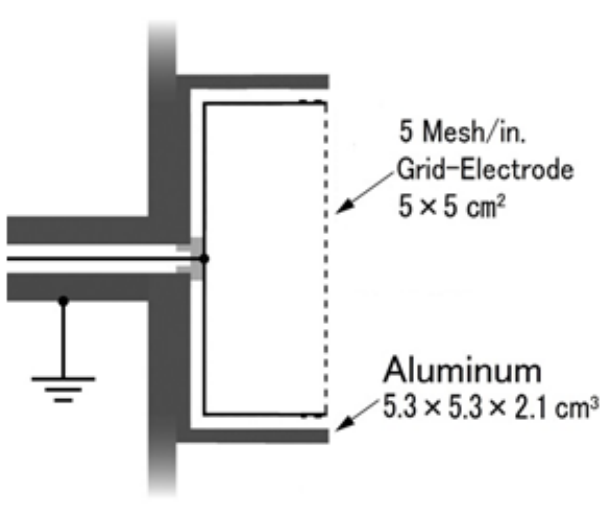

Figure 2. Details of the rectangular selfbiased RF electrode with a mesh grid.

on a display of the computer using the software that we have created using Microsoft Visual C++. The measurement method of plasma parameters mentioned above is explained by Ref. [24] in detail. The origin of $x$-axis is set at the wall ( $x=0$ $\mathrm{cm})$ of the cubic-box chamber, as shown in Fig. 1. The probe is fixed at the center of the cubic chamber $(x=7.5 \mathrm{~cm})$. In the case of Argon gas, plasma parameters generated by using electrode shown in Fig. 2 are almost the same as those produced by a grid-cage electrode [16].

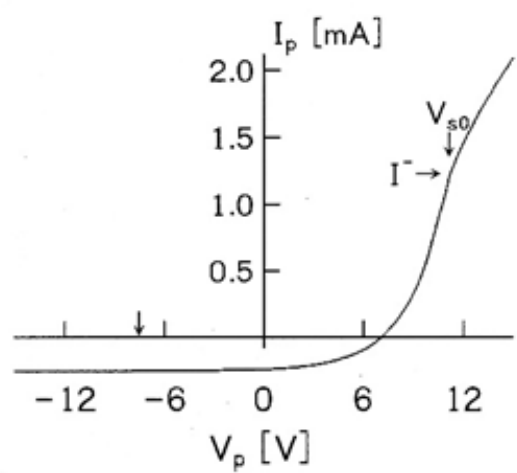

Figure 4. Time-averaged $I_{\mathrm{p}}-V_{\mathrm{p}}$ probe characteristic curve in $\mathrm{H}_{2}$ plasma. $P_{\mathrm{RF}}$ and $P_{\mathrm{H}_{2}}$ are $60 \mathrm{~W}$ and $0.9 \mathrm{~Pa}$, respectively. $x=7.5 \mathrm{~cm}$.

wire diameter of the electrode are 5 mesh/inch and $0.5 \mathrm{~mm}$, respectively. From a semi-log plot of this electron current $I_{\mathrm{e}}$, the space potential $V_{\mathrm{s} 0}=7.0 \mathrm{~V}$, electron saturation current $I_{\mathrm{es}}=2.05 \mathrm{~mA}$, electron temperature $T_{\mathrm{e}}=0.31 \mathrm{eV}$, and electron density 
$n_{\mathrm{e}}=2.1 \times 10^{10} \mathrm{~cm}^{-3}$ are drived. The method for obtaining these parameters has been already reported in Ref. [24]. The electron temperature $T_{\mathrm{e}}=0.31 \mathrm{eV}$ is too low to evolve the process of $\mathrm{H}_{2}^{*}(\mathrm{v})+\mathrm{e}_{l} \rightarrow \mathrm{H}^{-}+\mathrm{H}$ [15]. Therefore, negative hydrogen ions are supposed not to be produced. In this case, negative component of the probe current $I^{-}=2.02 \mathrm{~mA}$ seemes to express the electron suration current $I_{\mathrm{es}}$. With a use of the ion current

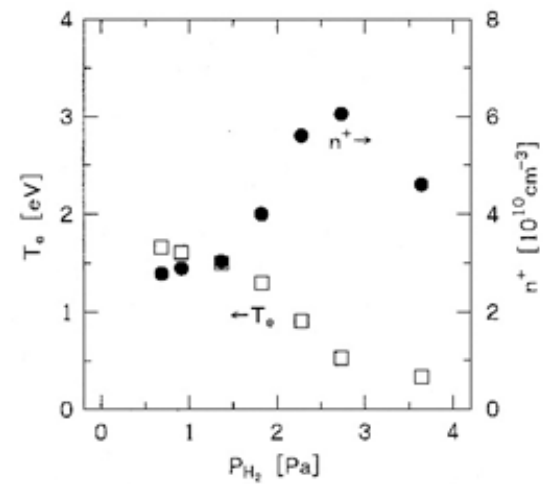

Figure 5. Variations of $T_{\mathrm{e}}$ and $n^{+}$as a function of hydrogen pressure $P_{\mathrm{H}_{2}}$ at $P_{\mathrm{RF}}=60 \mathrm{~W} . x=7.5 \mathrm{~cm}$.

Time averaged probe $I_{\mathrm{p}}-V_{\mathrm{p}}$ characteristic for pure hydrogen plasma in the case of $P_{\mathrm{RF}}=60 \mathrm{~W}$ and $P_{\mathrm{H}_{2}}=0.9 \mathrm{~Pa}$ is shown in Fig. 4 . From a semi-log plot of this electron current $I_{\mathrm{e}}$ one can obtain the space potential $V_{\mathrm{s} 0}=11.6 \mathrm{~V}$, electron temperature $T_{\mathrm{e}}=1.59 \mathrm{eV}$ and electron density $n_{\mathrm{e}}=6.27 \times 10^{9} \mathrm{~cm}^{-3}$. Here, $I^{-} / I^{+}=5.0$ (<28.5) from $I^{-}=1.25 \mathrm{~mA}$ and $I^{+}=0.25 \mathrm{~mA}$. This result suggests a generation of negative ions. Therefore, the above $n_{\mathrm{e}}$ does not express accurate electron density. By substituting $I_{\text {is }}=I^{+}=0.25$ $\mathrm{mA}$ and $T_{\mathrm{e}}=1.59 \mathrm{eV}$ into Eq. (5), $n_{\mathrm{i}}=n^{+}=$ $3.0 \times 10^{10} \mathrm{~cm}^{-3}$ is obtained. Further, by substitutng $I^{-} / I^{+}=5.0$ into Eq. (4), $n^{-} / n^{+}=$

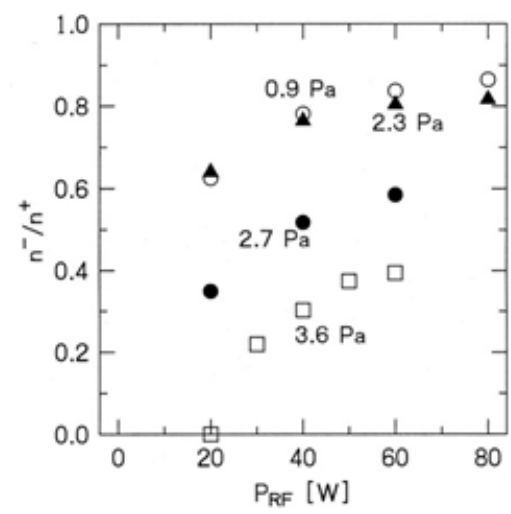

Figure 7. Variation of $n^{-} / n^{+}$as a function of RF power $P_{\mathrm{RF}}$ with gas pressure $P_{\mathrm{H}_{2}}$ as a parameter. $x=7.5 \mathrm{~cm}$.
$I^{+}=0.07 \mathrm{~mA}$ at $0.52 \mathrm{~V}$, indicated by a downward arrow in Fig. 3, one can obtain $I^{-} / I^{+}=$ $2.02 / 0.07=28.9$. Here, $I^{+}$is the positive ion sturation current $I_{\text {is }}$. We obtain $n^{-} / n^{+} \approx 0$ from eq. (4). This means that almost no negative hydrogen ions are generated in such a very low electron temperature of $0.31 \mathrm{eV}$ in Fig. 3.

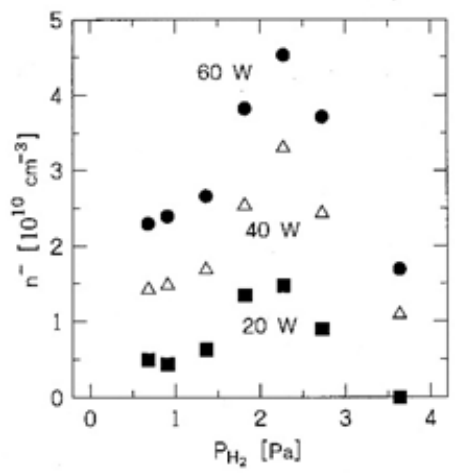

Figure 6. Dependence of $n^{-}$on $P_{\mathrm{H}_{2}}$ for three different RF powers. $x=7.5 \mathrm{~cm}$.

0.84 is obtained. The result suggests an efficient production of negative hydrogen ions.

Figure 5 shows the dependences of $T_{\mathrm{e}}$ and $n^{+}$on hydrogen pressure $P_{\mathrm{H}_{2}}$ at the center of the device. RF power $P_{\mathrm{RF}}$ is $60 \mathrm{~W}$. Here, $n^{+}$is obtained from Eq. (5). As shown clearly, $n^{+}$ increases first with an increase in $P_{\mathrm{H}_{2}}$, then reaches the maximum $6.1 \times 10^{10} \mathrm{~cm}^{-3}$, then decreases drastically. We can notice an efficient generation of positive hydrogen ions at $P_{\mathrm{H}_{2}}=2.7 \mathrm{~Pa}$. It is also noticed that $T_{\mathrm{e}}$ is lower than that of usual RF discharge plasmas [25]. The electron temperature monotonously decreases from $1.7 \mathrm{eV}$ to $0.3 \mathrm{eV}$ with an increase in $P_{\mathrm{H}_{2}}$ from $0.7 \mathrm{~Pa}$ to 3.6 Pa.

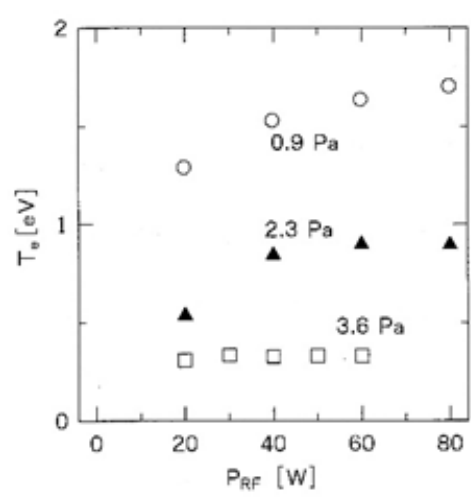

Figure 8. Variation of $T_{\mathrm{e}}$ as a function of RF power $P_{\mathrm{RF}}$ with gas pressure $P_{\mathrm{H}_{2}}$ as a parameter. $x=7.5 \mathrm{~cm}$. 
The dependence of $n^{-}$on $P_{\mathrm{H}_{2}}$ for three different RF powers at the center of the device is shown in Fig. 6. $n^{-}$reaches the maximum $4.6 \times$ $10^{10} \mathrm{~cm}^{-3}$ at $P_{\mathrm{H}_{2}}=2.3 \mathrm{~Pa}$, being almost independent of the RF power, where an efficient generation of negative ions occurs. This $n^{-}$is as the same level as the density obtained by using DC discharge with magnetic filter at $4 \mathrm{~kW}$ [2]. This result suggests that much higher density $n^{-}$plasma can be produced if parmanent magnets are employed [11]. In this case, the generation of highdensity hydrogen plasma $\left(n^{+}>1 \times 10^{11} \mathrm{~cm}^{-3}\right)$ could be possible around $T_{\mathrm{e}} \approx 1 \mathrm{eV}$ in the pressure range $0.9 \mathrm{~Pa}-2.3 \mathrm{~Pa}$ by performing a low power $(\approx$ 100W) RF discharge [17]. The dependences of $n^{-} / n^{+}$and $T_{\mathrm{e}}$ on RF power $P_{\mathrm{RF}}$ are shown in Figs. 7 and 8, respectively, with hydrogen pressure $P_{\mathrm{H}_{2}}$ as a parameter. With an increase in the RF power, the ratio $n^{-} / n^{+}$increases first, then it is saturated finally. This might be due to an increase in $\mathrm{H}_{2}^{*}(v)$ by an increase in the high energy electron $\mathrm{e}_{h}$ in the source region. Further, as the hydrogen pressure decreases, $T_{\mathrm{e}}$ raises to an appropriate range of $1.3-$ $1.5 \mathrm{eV}$ for an effective formation of $\mathrm{H}^{-}$ions. From Fig. 7 and Fig. 8 we can confirm that when $T_{\mathrm{e}}$ varies from $1.3 \mathrm{eV}$ to $1.74 \mathrm{eV}, n^{-} / n^{+}$increases from 0.79 to 0.87 . This temperature range almost agrees with the results derived in the numerical study [16]. The reason why generation efficiency of negative ions is so low at $P_{\mathrm{H}_{2}}=3.6 \mathrm{~Pa}$ might be due to that vibrationally excited molecules are not

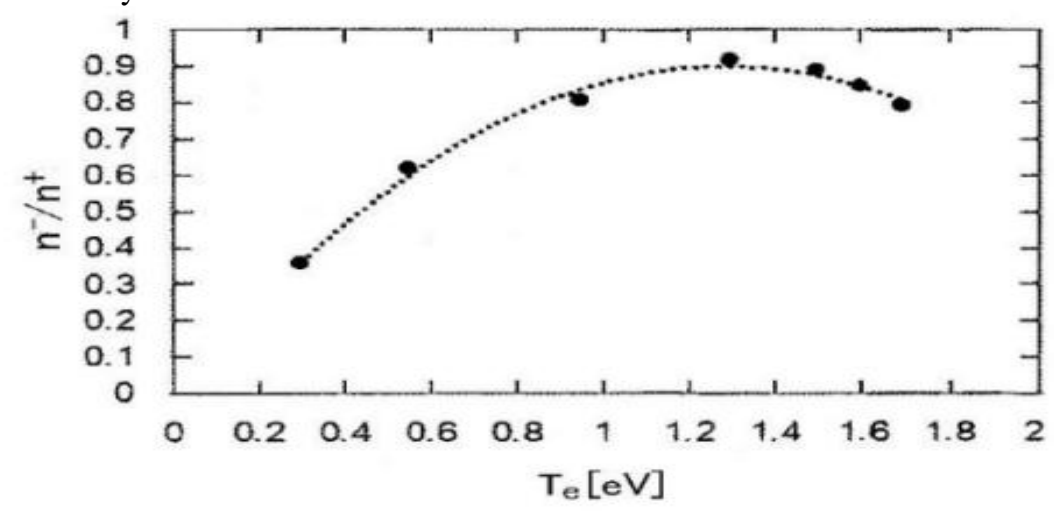

Figure 9. Diagram of $n^{-} / n^{+}$and $T_{\mathrm{e}}$, obtained from Figs. $5-8$ at $P_{\mathrm{RF}}$ $=60 \mathrm{~W}$. Dotted curve is derived from a second order polynomial approximation using least-squares method. enough supplied in such a low $T_{\mathrm{e}}$ plasma in the high pressure discharge.

\section{DISCUSSION}

In our method high $T_{\mathrm{e}}$ plasma is produced inside the grid electrode, where the reaction, $\mathrm{H}_{2}+\mathrm{e}_{h} \rightarrow \mathrm{H}_{2}^{*}(v)+\mathrm{e}$, takes place by the high electron temeparture electrons. On the other hand, in the region outside the grid electrode $T_{\mathrm{e}}$ decreases to a suitable level for the negative $\mathrm{H}^{-}$ion production through the reaction $\mathrm{H}_{2}^{*}(v)+\mathrm{e}_{l} \rightarrow$ $\mathrm{H}^{-}+\mathrm{H}$. The value of $T_{\mathrm{e}}$ can be basically varied by changing the mesh size under keeping the hydrogen pressure and input power. However, in this experiment, $T_{\mathrm{e}}$ is varied by a change of $P_{\mathrm{RF}}$ and/or $P_{\mathrm{H}_{2}}$ under the condition of fixed mesh size. The results are summarized by plotting all data on a $n^{-} / n^{+}-T_{\mathrm{e}}$ diagram, as shown in Fig. 9, with a use of the data at $P_{\mathrm{RF}}=60 \mathrm{~W}$ in Figs. $5-8$. The dotted curve is derived from a second order polynomial approximation using least-squares method. One can see that most suitable electron temperature for maximizing the ratio $n^{-} / n^{+}(\approx 0.9)$ is about $1.3 \mathrm{eV}$, being consistent with a numerical simulation [16]. The maximum value of $n^{-}=4.6 \times 10^{10} \mathrm{~cm}^{-3}$ is also established at $P_{\mathrm{H}_{2}}=2.3 \mathrm{~Pa}$ under $60 \mathrm{~W}$, as shown in Fig. 6, which is higher than the other result of $n^{-}=4.5 \times 10^{10} \mathrm{~cm}^{-3}$ at $P_{\mathrm{H}_{2}}=0.9 \mathrm{~Pa}$ under $P_{\mathrm{RF}}=4 \mathrm{~kW}$ [2]. 
Pulse-plasma operation [1] and magnetic filter method [3] are known as conventional production methods of negative hydrogen ions. However, in the former case the electron density fairly drops with a decrease in $T_{\mathrm{e}}$, accompanied by its temporal variation. Therefore, it is difficult to keep $T_{\mathrm{e}}$ fixed at most suitable electron temperature. In the latter case, it is difficult to suppress RF electric-field penetration into the downstream low $T_{\mathrm{e}}$ region, which disturbs negative ion production [13]. To overcome these faults, cesium is needed for seeding additionally to the discharge chamber to raise the ratio $n^{-} / n^{+}$[27]. Our method (i.e. selfbiased method) with a grid electrode is quite suitable for negative hydrogen ion production in RF plasmas, because the high and low electron temperature regions are separated by the grid electrode. The electron temperature outside the grid electrode can be controlled by the mesh size and discharge parameters. This method works even when the grid electrode is covered by resistive films such as hydrogenated amorphous films, diamond-like corbon, and so on. In Ar plasma the electron temperature can be further lowered by employing a fine mesh electrode, accompanied by an increase in the electron density.

\section{CONCLUSION}

We have demonstrated a new method with a use of a self-biased grid electrode for lowering electron temperature in an RF discharge plasma. High electron temperature plasma is produced inside the grid electrode for $\mathrm{H}_{2}^{*}(v)$ production. In the region outside the grid electrode the electron temperature decreases to a suitable value for a negative $\mathrm{H}^{-}$ion production through the dissociative attachment of $\mathrm{H}_{2}^{*}(v)$. In the case of mesh electrode of 5 mesh per inch, $n^{-} / n^{+}$reached to 0.87 at $P_{\mathrm{H}_{2}}=0.9 \mathrm{~Pa}$ and $P_{\mathrm{RF}}=80 \mathrm{~W}$ at the center of the discharge chamber. The maximum value of $n^{-}=4.6 \times 10^{10} \mathrm{~cm}^{-3}$ is established at $P_{\mathrm{H}_{2}}=2.3 \mathrm{~Pa}$ when the RF power is $60 \mathrm{~W}$. It is confirmed that negative hydrogen ions are produced efficiently in the range $T_{\mathrm{e}}=1.0-1.6 \mathrm{eV}$, which is consistent with a simulation result. The self-biased method with a grid electrode is quaite suitable to improve negative ion voluum generation in capacitively-coupled RF discharges.

\section{REFERENCES}

[1]. Samukawa, S. Sakamoto, K. and Ichiki, K. (2002) Generating high-efficiency neutral beams by using negative ions in an inductively coupled plasma source. Journal of Vacuum Science and Technology. A20(5), 1566-1573.

[2]. Eenshuistra, P.J. Gochitashvilli, M. Becker, R. Klein, A.W. and Hopman, H.J. (1990) Negative hydrogen ion densities and drift velocities in a multicusp ion source. Journal of Applied Physics. 67, 85-96.
[3]. Stamate, E. and Draghici, M. (2012) High electronegativity multidipolor electron cycrotron resonance plasma source for eching by negative ions. Journal of Applied Physics. 111, 083303-1-083303-6.

[4]. Hopkins, M.B. and Mellon, K.N. (1991) Enhanced production of negative ions in low-pressure hydrogen and duetrium discharges. Physical Review Letters. 67, 449-452.

[5]. Kato, K. Iizuka, S. and Sato, N. (1994) Electrontemperature control for plasmas passing through a negatively biased grid. Applied Physics Letters. 76, 816-818.

[6]. Iizuka, S. Koizumi, T. Takada, T. and Sato, N. (1993) Effect of electron temperature on negative hydrogen ion production in a low-pressure Ar discharge plasma with methane. Applied Physics Letters. 63(12), 1619-1621.

[7]. Hong, J.I. Seo, S.H. Kim, S.S. Yoon, N.S. Chang, C. S. and Chang, H.Y. (1999) Electron temperature control with grid bias in inductively coupled argon plasma. Physics of Plasmas. 6, 1017-1028.

[8]. Kato, K. Iizuka, S. and Sato, N. (2000) Electron temperature control by varying size of slits made in a grid. Applied Physics Letters. 76, 547-579.

[9]. Kato, K. Emi, J. and Iizuka, S. (2008) Control of electron temperature by varying DC voltage to a mesh grid branketed with thin film in plasmas. Japanese Journel of Applied Physics. 47, 8565-8569.

[10]. Kato, K. lizuka, S. Ganguly, G. Ikeda, T. Matsuda, A. and Sato, N. (1997) Electron and ion energy controls in a radio frquency discharge plasma with silane. Japanese Journel of Applied Physics. 36, Part 1, No. 7B 45474550 .

[11]. Iizuka, S. Kato, K. Takahashi, A. Nakagomi, K. and Sato, N. (1997) Negative hydrogen ions produced by electron temperature control in an RF plasma. Japanese Journel of Applied Physics. 36, Part. 1, No. 7B 45514553 .

[12]. Jyobira, Y. Ito, D. and Fukumasa, O. (2008) Enhancement of pure volume negative ion production using a grid bias method or a magnetic filter method. Review of Scientific Instument. 79, 02A508-1-02A5084.

[13]. Okada, J. Nakao, Y. Tauchi, Y. and Fukumasa, O. (2008) Efficient negative ion production in rf plasmas using grid bias method. Review Scientific Instrument. 79, 02A502-1-02A502-3.

[14]. Shimizu, T. Iizuka, S. Kato, K. and Sato, N. (2003) High quality diamond formation by electron temperature control in methane-hydrogen plasma. Plasma Sources Sciense and Technology. 12, S21-S27.

[15]. Hiskes, J.R. and Karo, A.M. (1984) Generation of negative ions in tandem high density hydrogen discharges, Japanese Journal of Applied Physics. 56, 1927-1938.

[16]. Fukumasa., O. and Ohashi, S. (1989) Numarical study on tandem source for production of negative ions. Journal of Physics. D: Applied Physics. 22, 1931-1934.

[17]. Kato, K. Gohda, T. and Iizuka, S. (2016) Production of low electron-temperature and high density plasma by using a grid-cage electrode for RF discharge. Journal of Scientific Research \& Reports. 11(2), 1-9.

[18]. Hatta, Y. Gas Discharges. Published by Kindai Kagakusha, (Tokyo, 1996) p. 129.

[19]. Tonks, L. and Langmuir, I. (1929) A general theory of the plasma of an arc. Physical Review. 34, 876-922.

[20]. Boschi, A. and Magistrelli, F. (1963) Effect of a R.F. signal on the characteristic of a Langmuir probe. IL. NUOVO CIMENTO. 29, 487-499.

[21]. Holmes, A.J.T. Lea, A.L.M. Newman, F. and Nightingale, M.P.S. (1987) Extraction of $\mathrm{H}^{-}$and $\mathrm{D}^{-}$ ions from a large magnetic multipole source. Review Scientific Instrument. 58, 223-234. 
[22]. Coburn, J.W. Kay, E. (1972) Positive ion bombardment of substrates in rf diode glow discharge sputtering. J. Applied Physics. 43, 4965-4971.

[23]. Kohler, K. Coburn J.W. Home D.E. and Kay, E. (1985) Plasma potentials of $13.56 \mathrm{MHz}$ rf argon glow discharges in a planar system. Journal of Applied Physics. 57, 59-66.

[24]. Kato, K. and Iizuka, S. (2016) Analysis of Langmuir probe characteristics for measurement of plasma parameters in RF discharge plasmas. Journal of Applied Mathematics and Physics. 4, 1811-1836.

[25]. Gojyak, V.A. (2011) Electical and plasma parameters of ICP with high coupling efficiency. Plasma Sources Science and Technology. 20, 025004-1-025004-2.

[26]. Wong, A.Y. Mamas, D.L. and Arnush, D. (1975) Negative ion plasmas. Physics of Fluids. 18, 1489-1493.

[27]. Ando, A. Tsumori, K. Kaneko, O. Takeiri, Y. Asano, E. Kawamoto, T. Akiyama, R. and Kuroda, T. (1994) Large current negative hydrogen ion beam production. Physics of Plasmas. 1, 2813-2815. 